\title{
Foundations of Conic Conformal Geometric Algebra and Compact Versors for Rotation, Translation and Scaling
}

\author{
Eckhard Hitzer and Stephen J. Sangwine
}

\begin{abstract}
This paper explains in algebraic detail how two-dimensional conics can be defined by the outer products of conformal geometric algebra (CGA) points in higher dimensions. These multivector expressions code all types of conics in arbitrary scale, location and orientation. Conformal geometric algebra of two-dimensional Euclidean geometry is fully embedded as an algebraic subset. With small model preserving modifications, it is possible to consistently define in conic CGA versors for rotation, translation and scaling, similar to [10], but simpler, especially for translations.
\end{abstract}

Mathematics Subject Classification (2010). Primary 15A66; Secondary 11E88, 15A15, 15A09.

Keywords. Clifford algebra, conformal geometric algebra, conics, versors.

\section{Introduction}

Two dimensional conics can be represented in extended conformal geometric algebras (CGA) in several ways. Historically, [12] managed to express conics in Grassmann algebra and with the use of a tensor product as a 10th order product of five points on the conic. The method we study here in algebraic detail was first outlined by C. Perwass in Chapter 4.5 of [13] and uses only a simple fifth order product of the five contact points. Most recently double CGA (DCGA)[6] and quadric CGA (QCGA)[1] have been proposed ${ }^{1}$ in this context. We also compare with a recent modified approach provided in [10]. We first focus on the rational algebraic core structure of conic conformal geometric algebra (CCGA) to be presented in some detail. While doing that, we introduce a small model preserving modification in the basic algebraic definitions, that is key to preserving the translation formulation of

${ }^{1}$ For further literature see the references in [1]. 
[13], but allows to consistently keep the full system of versors for rotations, translations and scaling. This formulation of the translation versors is simpler than the formulation given in [10].

The paper is structured as follows. Section 2 defines CCGA as an extension of CGA, and provides a detailed set of important algebraic relations computed for CCGA. Section 3 shows how points and all two-dimensional CGA objects are defined and embedded in CCGA. Section 4 defines blade expressions for conics and axis aligned conics in CCGA. Section 5 explains how to compute intersections, and Section 6 formulates versors for rotations, translations and scaling. The paper ends with a conclusion in Section 7 followed by acknowledgments and references.

\section{CCGA definition}

This section introduces CCGA. We specify its basis vectors in a slightly modified way, and show important blade computations. We use the following notation: Lower-case bold letters denote basis blades and multivectors (vector or multivector a). Italic lower-case letters refer to multivector components $\left(a_{1}, x, y^{2}, \cdots\right)$. For example, $a_{i}$ is the $i^{t h}$ coordinate of the (multi)vector a. Constant scalars are denoted using lower-case default text font (constant radius r) or simply $r$. The superscript star used in $\mathbf{x}^{*}$ represents the dualization of the multivector $\mathbf{x}$. Finally, subscript $\varepsilon$ on $\mathbf{x}_{\varepsilon}$ refers to the two-dimensional Euclidean vector associated to the vector $\mathbf{x}$ of CCGA.

Note that when used in geometric algebra the inner product, contractions and the outer product take precedence over the full geometric product. For instance, $\mathbf{a} \wedge \mathbf{b I}=(\mathbf{a} \wedge \mathbf{b}) \mathbf{I}, \mathbf{a} \cdot \mathbf{b I}=(\mathbf{a} \cdot \mathbf{b}) \mathbf{I}, \mathbf{a}\rfloor \mathbf{b I}=(\mathbf{a}\rfloor \mathbf{b}) \mathbf{I}$, and $\mathbf{a}\lfloor\mathbf{b I}=(\mathbf{a}\lfloor\mathbf{b}) \mathbf{I}$, etc.

\subsection{CCGA basis and metric}

The algebraic equations in this section can be either computed by hand, expanding all blades in terms of basis vectors, or they can be computed with software, like the Clifford Multivector Toolbox (for MATLAB)[14]. The current section is perhaps more detailed than a first time reader may need to generally comprehend the model we present. A first time reader might therefore simply look at the definitions in (1) to (14), and (37) to (39), and only come back to the other relationships listed in this section, if he wants to verify symbolic computations in the rest of this paper.

The CCGA $C l(5,3)$ is defined over the 8 -dimensional real vector space $\mathbb{R}^{5,3}$. The basis vectors of the space ${ }^{2}$ can be divided into three groups: $\left\{\mathbf{e}_{1}, \mathbf{e}_{2}\right\}$ (corresponding to the Euclidean vectors of $\mathbb{R}^{2}$ ), and six null vectors $\left\{\mathbf{e}_{o 1}, \mathbf{e}_{o 2}, \mathbf{e}_{o 3}\right\}$, and $\left\{\mathbf{e}_{\infty 1}, \mathbf{e}_{\infty 2}, \mathbf{e}_{\infty 3}\right\}$. The inner products between them are defined in Table 1 .

For efficient computation, a diagonal metric matrix may often be useful. The algebra $C l(5,3)$ generated by the Euclidean basis $\left\{\mathbf{e}_{1}, \mathbf{e}_{2}\right\}$, and three basis vectors

\footnotetext{
${ }^{2}$ We use for null vectors the notation $\mathbf{e}_{o}$, and $\mathbf{e}_{\infty}$ with added indexes $1,2,3$, because this intuitive notation for CGA null vectors became widespread with [4], replacing the earlier notation $\bar{n}$ and $n$. The notation $\bar{n}$ and $n$ with added indexes 1,2,3 was used in [13], but [1] consistently combined instead $\mathbf{e}_{o}$, and $\mathbf{e}_{\infty}$ with added indexes 1,2,3, etc. Avoiding the introduction of further new conventions, we adopt the notation for basis vectors following [1].
} 
TABLE 1. Inner products between CCGA basis vectors.

\begin{tabular}{r|cccccccc} 
& $\mathbf{e}_{1}$ & $\mathbf{e}_{2}$ & $\mathbf{e}_{o 1}$ & $\mathbf{e}_{\infty 1}$ & $\mathbf{e}_{o 2}$ & $\mathbf{e}_{\infty 2}$ & $\mathbf{e}_{o 3}$ & $\mathbf{e}_{\infty 3}$ \\
\hline $\mathbf{e}_{1}$ & 1 & 0 & $\cdot$ & $\cdot$ & $\cdot$ & $\cdot$ & $\cdot$ & $\cdot$ \\
$\mathbf{e}_{2}$ & 0 & 1 & $\cdot$ & $\cdot$ & $\cdot$ & $\cdot$ & $\cdot$ & $\cdot$ \\
$\mathbf{e}_{o 1}$ & $\cdot$ & $\cdot$ & 0 & -1 & $\cdot$ & $\cdot$ & $\cdot$ & $\cdot$ \\
$\mathbf{e}_{\infty 1}$ & $\cdot$ & $\cdot$ & -1 & 0 & $\cdot$ & $\cdot$ & $\cdot$ & $\cdot$ \\
$\mathbf{e}_{o 2}$ & $\cdot$ & $\cdot$ & $\cdot$ & $\cdot$ & 0 & -1 & $\cdot$ & $\cdot$ \\
$\mathbf{e}_{\infty 2}$ & $\cdot$ & $\cdot$ & $\cdot$ & $\cdot$ & -1 & 0 & $\cdot$ & $\cdot$ \\
$\mathbf{e}_{o 3}$ & $\cdot$ & $\cdot$ & $\cdot$ & $\cdot$ & $\cdot$ & $\cdot$ & 0 & -1 \\
$\mathbf{e}_{\infty 3}$ & $\cdot$ & $\cdot$ & $\cdot$ & $\cdot$ & $\cdot$ & $\cdot$ & -1 & 0
\end{tabular}

$\left\{\mathbf{e}_{+1}, \mathbf{e}_{+2}, \mathbf{e}_{+3}\right\}$ squaring to +1 along with three other basis vectors $\left\{\mathbf{e}_{-1}, \mathbf{e}_{-2}, \mathbf{e}_{-3}\right\}$ squaring to -1 would correspond to a diagonal metric matrix.

Relating the diagonal two-dimensional Minkowski plane basis $\left\{\mathbf{e}_{+i}, \mathbf{e}_{-i}\right\}$ to the null basis $\left\{\mathbf{e}_{o i}, \mathbf{e}_{\infty i}\right\}$, for $i=1,2$ or 3 , is unique up to three non-zero parameters $\lambda_{i} \in \mathbb{R} \backslash\{0\}$,

$$
\mathbf{e}_{\infty i}=\frac{\lambda_{i}}{\sqrt{2}}\left(\mathbf{e}_{+i}+\mathbf{e}_{-i}\right), \quad \mathbf{e}_{o i}=\frac{1}{\lambda_{i} \sqrt{2}}\left(\mathbf{e}_{-i}-\mathbf{e}_{+i}\right) .
$$

The parameters ${ }^{3} \lambda_{i}, i=1,2,3$, are usually in conformal geometric algebra of the plane, or in the modeling of conics in $C l(5,3)$ by Perwass [13] or by Hrdina et al [10], simply all set to $\lambda_{1}=\lambda_{2}=\lambda_{3}=\sqrt{2}$. In contrast to that, e.g., El Mir et al [5] have exploited this degree of freedom in conformal geometry for an elegant new formulation of viewpoint change representation. By reasons, that will become evident during the course of this paper, we decide to rather choose the symmetric values $\lambda_{1}=\lambda_{2}=\lambda_{3}=1$.

The transformation from the diagonal metric basis to that of Table 1 can now be defined as follows: for $1 \leq i, j \leq 3$,

$$
\mathbf{e}_{\infty i}=\frac{1}{\sqrt{2}}\left(\mathbf{e}_{+i}+\mathbf{e}_{-i}\right), \quad \mathbf{e}_{o i}=\frac{1}{\sqrt{2}}\left(\mathbf{e}_{-i}-\mathbf{e}_{+i}\right) .
$$

We further define for later use another pair of null vectors

$$
\mathbf{e}_{\infty}=\frac{1}{2}\left(\mathbf{e}_{\infty 1}+\mathbf{e}_{\infty 2}\right), \quad \mathbf{e}_{o}=\mathbf{e}_{o 1}+\mathbf{e}_{o 2} .
$$

Inner products lead to

$$
\begin{aligned}
& \mathbf{e}_{\infty i} \cdot \mathbf{e}_{o i}=-1, \quad \mathbf{e}_{\infty} \cdot \mathbf{e}_{o}=-1, \quad \mathbf{e}_{o}^{2}=\mathbf{e}_{\infty}^{2}=0, \\
& \mathbf{e}_{\infty 1} \cdot \mathbf{e}_{o}=\mathbf{e}_{\infty 2} \cdot \mathbf{e}_{o}=-1, \quad \mathbf{e}_{\infty 3} \cdot \mathbf{e}_{o}=0, \quad \mathbf{e}_{\infty i} \cdot \mathbf{e}_{\infty}=0,
\end{aligned}
$$

We further define the bivectors $E_{i}, E$, as

$$
E_{i}=\mathbf{e}_{\infty i} \wedge \mathbf{e}_{o i}=\mathbf{e}_{+i} \mathbf{e}_{-i}, \quad E=\mathbf{e}_{\infty} \wedge \mathbf{e}_{o},
$$

\footnotetext{
${ }^{3}$ The parameters $\lambda_{i}, i=1,2,3$, parameterize a continuous set of horospheres [5].
} 
and obtain the following products

$$
\begin{gathered}
E_{i}^{2}=1, \quad E_{i} E_{j}=E_{j} E_{i}, \\
\mathbf{e}_{o i} E_{i}=-E_{i} \mathbf{e}_{o i}=-\mathbf{e}_{o i}, \quad \mathbf{e}_{\infty i} E_{i}=-E_{i} \mathbf{e}_{\infty i}=\mathbf{e}_{\infty i}, \\
\mathbf{e}_{o j} E_{i} \stackrel{i \neq i}{=} E_{i} \mathbf{e}_{o j}, \quad \mathbf{e}_{\infty j} E_{i} \stackrel{i \neq i}{=} E_{i} \mathbf{e}_{\infty j}, \\
E^{2}=1, \quad \mathbf{e}_{o} E=-E \mathbf{e}_{o}=-\mathbf{e}_{o}, \quad \mathbf{e}_{\infty} E=-E \mathbf{e}_{\infty}=\mathbf{e}_{\infty} .
\end{gathered}
$$

For brevity of notation and clarity, we also define the following blades:

$$
\begin{aligned}
\mathbf{I}_{\infty 12} & =\mathbf{e}_{\infty 1} \mathbf{e}_{\infty 2}, \quad \mathbf{I}_{\infty}=\mathbf{I}_{\infty 12} \mathbf{e}_{\infty 3}, \quad \mathbf{I}_{o 12}=\mathbf{e}_{o 1} \mathbf{e}_{o 2}, \quad \mathbf{I}_{o}=\mathbf{I}_{o 12} \mathbf{e}_{o 3}, \\
\mathbf{I}_{\infty o} & =\mathbf{I}_{\infty} \wedge \mathbf{I}_{o}=-E_{1} E_{2} E_{3}, \\
\mathbf{e}_{\infty 12}^{\triangleright} & =\mathbf{e}_{\infty 1}-\mathbf{e}_{\infty 2}, \quad \mathbf{I}_{\infty}^{\triangleright}=\mathbf{e}_{\infty 12}^{\triangleright} \mathbf{e}_{\infty 3}, \quad \mathbf{e}_{o 12}^{\triangleright}=\mathbf{e}_{o 1}-\mathbf{e}_{o 2}, \quad \mathbf{I}_{o}^{\triangleright}=\mathbf{e}_{o 12}^{\triangleright} \mathbf{e}_{o 3}, \\
\mathbf{I}^{\triangleright} & =\mathbf{I}_{\infty}^{\triangleright} \wedge \mathbf{I}_{o}^{\triangleright} .
\end{aligned}
$$

We note that

$$
\begin{gathered}
\mathbf{I}_{\infty o}^{2}=1, \quad \mathbf{I}_{o} \mathbf{I}_{\infty o}=-\mathbf{I}_{\infty o} \mathbf{I}_{o}=\mathbf{I}_{o}, \quad \mathbf{I}_{\infty} \mathbf{I}_{\infty o}=-\mathbf{I}_{\infty o} \mathbf{I}_{\infty}=-\mathbf{I}_{\infty}, \\
\left(\mathbf{e}_{\infty 12}^{\triangleright} \wedge \mathbf{e}_{o 12}^{\triangleright}\right)^{-1}=\frac{1}{4} \mathbf{e}_{\infty 12}^{\triangleright} \wedge \mathbf{e}_{o 12}^{\triangleright}, \quad \mathbf{I}_{\infty 12} \wedge \mathbf{I}_{o 12}=-E_{1} E_{2}, \\
\mathbf{I}^{\triangleright}=-\mathbf{e}_{\infty 12}^{\triangleright} \wedge \mathbf{e}_{o 12}^{\triangleright} E_{3}, \quad\left(\mathbf{I}^{\triangleright}\right)^{2}=\left(\mathbf{e}_{\infty 12}^{\triangleright} \wedge \mathbf{e}_{o 12}^{\triangleright}\right)^{2}=4, \quad\left(\mathbf{I}^{\triangleright}\right)^{-1}=\frac{1}{4} \mathbf{I}^{\triangleright} \\
\left.\mathbf{I}_{\infty}^{\triangleright} \cdot \mathbf{I}_{o}^{\triangleright}=\mathbf{I}_{o}^{\triangleright} \cdot \mathbf{I}_{\infty}^{\triangleright}=\mathbf{I}_{\infty}^{\triangleright}\right\rfloor \mathbf{I}_{o}^{\triangleright}=\mathbf{I}_{\infty}^{\triangleright}\left\lfloor\mathbf{I}_{o}^{\triangleright}=-2 .\right.
\end{gathered}
$$

Note, that in the last line, the product symbols $\rfloor$ and $\lfloor$ express the left- and right contraction, respectively. We have the following outer product relationships

$$
\begin{aligned}
\mathbf{I}_{\infty 12} & =-\mathbf{e}_{\infty 1} \wedge \mathbf{e}_{\infty 12}^{\triangleright}=-\mathbf{e}_{\infty 2} \wedge \mathbf{e}_{\infty 12}^{\triangleright}=-\mathbf{e}_{\infty} \wedge \mathbf{e}_{\infty 12}^{\triangleright} \\
& =-\mathbf{e}_{\infty 1} \mathbf{e}_{\infty 12}^{\triangleright}=-\mathbf{e}_{\infty 2} \mathbf{e}_{\infty 12}^{\triangleright}=-\mathbf{e}_{\infty} \mathbf{e}_{\infty 12}^{\triangleright}, \\
\mathbf{I}_{o 12} & =-\mathbf{e}_{o 1} \wedge \mathbf{e}_{o 12}^{\triangleright}=-\mathbf{e}_{o 2} \wedge \mathbf{e}_{o 12}^{\triangleright}=-\frac{1}{2} \mathbf{e}_{o} \wedge \mathbf{e}_{o 12}^{\triangleright} \\
& =-\mathbf{e}_{o 1} \mathbf{e}_{o 12}^{\triangleright}=-\mathbf{e}_{o 2} \mathbf{e}_{o 12}^{\triangleright}=-\frac{1}{2} \mathbf{e}_{o} \mathbf{e}_{o 12}^{\triangleright}, \\
\mathbf{I}_{\infty 12} \wedge \mathbf{I}_{o 12} & =-E_{1} \wedge \mathbf{e}_{\infty 12}^{\triangleright} \wedge \mathbf{e}_{o 12}^{\triangleright}=-E_{2} \wedge \mathbf{e}_{\infty 12}^{\triangleright} \wedge \mathbf{e}_{o 12}^{\triangleright} \\
& =-\frac{1}{2} E \wedge \mathbf{e}_{\infty 12}^{\triangleright} \wedge \mathbf{e}_{o 12}^{\triangleright}=-\frac{1}{2} E \mathbf{e}_{\infty 12}^{\triangleright} \wedge \mathbf{e}_{o 12}^{\triangleright} .
\end{aligned}
$$

And we have the following inner products $(i=1,2)$

$$
\begin{aligned}
& \mathbf{e}_{o 12}^{\triangleright}=2 \mathbf{e}_{\infty} \cdot \mathbf{I}_{o 12}, \quad \mathbf{I}_{o}^{\triangleright}=2 \mathbf{e}_{\infty} \cdot \mathbf{I}_{o}, \quad \mathbf{e}_{\infty 12}^{\triangleright}=\mathbf{e}_{o} \cdot \mathbf{I}_{\infty 12}, \quad \mathbf{I}_{\infty}^{\triangleright}=\mathbf{e}_{o} \cdot \mathbf{I}_{\infty}, \\
& \left(\mathbf{e}_{o i} \cdot \mathbf{I}_{\infty}\right) \cdot \mathbf{I}_{o}=\mathbf{e}_{o i}, \quad\left(\mathbf{e}_{\infty i} \cdot \mathbf{I}_{o}\right) \cdot \mathbf{I}_{\infty}=\mathbf{e}_{\infty i}, \\
& \left(\mathbf{e}_{o} \cdot \mathbf{I}_{\infty}\right) \cdot \mathbf{I}_{o}=\mathbf{e}_{o}, \quad\left(\mathbf{e}_{\infty} \cdot \mathbf{I}_{o}\right) \cdot \mathbf{I}_{\infty}=\mathbf{e}_{\infty} \\
& \mathbf{e}_{\infty} \cdot \mathbf{I}_{\infty o}=-\frac{1}{2} \mathbf{I}_{\infty} \wedge \mathbf{I}_{o}^{\triangleright}, \quad \mathbf{e}_{o} \cdot \mathbf{I}_{\infty O}=\mathbf{I}_{\infty}^{\triangleright} \wedge \mathbf{I}_{o}, \\
& \mathbf{e}_{\infty i} \cdot \mathbf{e}_{\infty 12}^{\triangleright}=0, \quad \mathbf{e}_{\infty i} \cdot \mathbf{I}_{\infty}^{\triangleright}=0, \quad \mathbf{e}_{\infty} \cdot \mathbf{e}_{\infty 12}^{\triangleright}=0, \quad \mathbf{e}_{\infty} \cdot \mathbf{I}_{\infty}^{\triangleright}=0, \\
& \mathbf{e}_{o i} \cdot \mathbf{e}_{o 12}^{\triangleright}=0, \quad \mathbf{e}_{o i} \cdot \mathbf{I}_{o}^{\triangleright}=0, \quad \mathbf{e}_{o} \cdot \mathbf{e}_{o 12}^{\triangleright}=0, \quad \mathbf{e}_{o} \cdot \mathbf{I}_{o}^{\triangleright}=0, \\
& \mathbf{e}_{\infty} \cdot \mathbf{e}_{o 12}^{\triangleright}=0, \quad \mathbf{e}_{\infty} \cdot \mathbf{I}_{o}^{\triangleright}=0, \quad \mathbf{e}_{o} \cdot \mathbf{e}_{\infty 12}^{\triangleright}=0, \quad \mathbf{e}_{o} \cdot \mathbf{I}_{\infty}^{\triangleright}=0, \\
& \mathbf{e}_{\infty} \cdot \mathbf{I}^{\triangleright}=0, \quad \mathbf{e}_{o} \cdot \mathbf{I}^{\triangleright}=0, \quad E \cdot \mathbf{I}^{\triangleright}=0 .
\end{aligned}
$$


As consequence we obtain

$$
\begin{aligned}
\mathbf{I}_{\infty} & =-\mathbf{e}_{\infty 1} \mathbf{I}_{\infty}^{\triangleright}=-\mathbf{e}_{\infty 1} \wedge \mathbf{I}_{\infty}^{\triangleright}=-\mathbf{e}_{\infty 2} \mathbf{I}_{\infty}^{\triangleright}=-\mathbf{e}_{\infty 2} \wedge \mathbf{I}_{\infty}^{\triangleright} \\
& =-\mathbf{e}_{\infty} \mathbf{I}_{\infty}^{\triangleright}=-\mathbf{e}_{\infty} \wedge \mathbf{I}_{\infty}^{\triangleright}, \\
\mathbf{I}_{\infty} \wedge \mathbf{I}_{o}^{\triangleright} & =-\mathbf{e}_{\infty 1} \wedge \mathbf{I}^{\triangleright}=-\mathbf{e}_{\infty 2} \wedge \mathbf{I}^{\triangleright}=-\mathbf{e}_{\infty} \wedge \mathbf{I}^{\triangleright}=-\mathbf{e}_{\infty} \mathbf{I}^{\triangleright}=-\mathbf{I}^{\triangleright} \mathbf{e}_{\infty}, \\
2 \mathbf{I}_{o} & =-\mathbf{e}_{o} \mathbf{I}_{o}^{\triangleright}=-\mathbf{e}_{o} \wedge \mathbf{I}_{o}^{\triangleright}, \quad-2 \mathbf{I}_{\infty}^{\triangleright} \wedge \mathbf{I}_{o}=\mathbf{e}_{o} \mathbf{I}^{\triangleright}=\mathbf{e}_{o} \wedge \mathbf{I}^{\triangleright}=\mathbf{I}^{\triangleright} \mathbf{e}_{o}, \\
2 \mathbf{I}_{\infty o} & =E \mathbf{I}^{\triangleright}=E \wedge \mathbf{I}^{\triangleright}=\mathbf{I}^{\triangleright} E, \quad \mathbf{I}^{\triangleright}=2 E \mathbf{I}_{\infty o}=2 \mathbf{I}_{\infty o} E .
\end{aligned}
$$

The last relationship means that the quadvector $\mathbf{I}^{\triangleright}$ can be obtained from the 6vector $\mathbf{I}_{{ }_{\infty} o}$ by left- or right division with bivector $E$, since $E^{-1}=E$. Similarly, $\mathbf{I}_{o}^{\triangleright}$ is obtained from $\mathbf{I}_{o}$ by factoring out $\mathbf{e}_{o}$ as in (30), (22), and $\mathbf{I}_{\infty}^{\triangleright}$ from $\mathbf{I}_{\infty}$ by factoring out $\mathbf{e}_{\infty}$ as in (32), (22). We can summarize for the multivector basis of the geometric algebra Minkowski plane spanned by $\left\{\mathbf{e}_{o}, \mathbf{e}_{\infty}\right\}$, and the quadvector $\mathbf{I}^{\triangleright}$ the important set of relations

$$
\left\{1, \mathbf{e}_{o}, \mathbf{e}_{\infty}, E\right\} \wedge \mathbf{I}^{\triangleright}=\left\{1, \mathbf{e}_{o}, \mathbf{e}_{\infty}, E\right\} \mathbf{I}^{\triangleright}=\mathbf{I}^{\triangleright}\left\{1, \mathbf{e}_{o}, \mathbf{e}_{\infty}, E\right\} .
$$

Similar relationships hold separately for products with the simple bivector $\mathbf{I}_{\infty}^{\triangleright}$, or with $\mathbf{I}_{o}^{\triangleright}$, respectively,

$$
\begin{aligned}
& \left\{1, \mathbf{e}_{o}, \mathbf{e}_{\infty}, E\right\} \wedge \mathbf{I}_{\infty}^{\triangleright}=\left\{1, \mathbf{e}_{o}, \mathbf{e}_{\infty}, E\right\} \mathbf{I}_{\infty}^{\triangleright}=\mathbf{I}_{\infty}^{\triangleright}\left\{1, \mathbf{e}_{o}, \mathbf{e}_{\infty}, E\right\}, \\
& \left\{1, \mathbf{e}_{o}, \mathbf{e}_{\infty}, E\right\} \wedge \mathbf{I}_{o}^{\triangleright}=\left\{1, \mathbf{e}_{o}, \mathbf{e}_{\infty}, E\right\} \mathbf{I}_{o}^{\triangleright}=\mathbf{I}_{o}^{\triangleright}\left\{1, \mathbf{e}_{o}, \mathbf{e}_{\infty}, E\right\} .
\end{aligned}
$$

We define the two-dimensional Euclidean pseudo-scalar $\mathbf{I}_{\mathcal{\varepsilon}}$ bivector in $\mathbb{R}^{2}$ :

$$
\mathbf{I}_{\varepsilon}=\mathbf{e}_{1} \mathbf{e}_{2}, \quad \mathbf{I}_{\varepsilon}^{2}=-1, \quad \mathbf{I}_{\varepsilon}^{-1}=-\mathbf{I}_{\varepsilon},
$$

and the conformal pseudo-scalar $\mathbf{I}_{\mathbf{C}}$ by

$$
\mathbf{I}_{\mathrm{C}}=\mathbf{e}_{1} \mathbf{e}_{2} \mathbf{e}_{\infty} \wedge \mathbf{e}_{o}=\mathbf{I}_{\mathcal{E}} E, \quad \mathbf{I}_{\mathrm{C}}^{2}=-1, \quad \mathbf{I}_{\mathrm{C}}^{-1}=-\mathbf{I}_{\mathrm{C}}
$$

The full pseudo-scalar $\mathbf{I}$ and its inverse $\mathbf{I}^{-1}$ (used for dualization) are:

$$
\mathbf{I}=\mathbf{I}_{\varepsilon} \mathbf{I}_{\infty o}=\frac{1}{2} \mathbf{I}_{C} \mathbf{I}^{\triangleright}=\frac{1}{2} \mathbf{I}_{\varepsilon} E \mathbf{I}^{\triangleright}=-\mathbf{I}_{\varepsilon} E_{1} E_{2} E_{3}, \quad \mathbf{I}^{2}=-1, \quad \mathbf{I}^{-1}=-\mathbf{I} .
$$

The dual of a multivector indicates division by the pseudo-scalar, e.g., $\mathbf{a}^{*}=-\mathbf{a I}$, $\mathbf{a}=\mathbf{a}^{*} \mathbf{I}$. From eq. (1.19) in [9], we have the useful duality between outer and inner products of non-scalar blades $A, B$ in geometric algebra:

$$
(A \wedge B)^{*}=A \cdot B^{*}, \quad A \wedge\left(B^{*}\right)=(A \cdot B)^{*} \Leftrightarrow A \wedge(B \mathbf{I})=(A \cdot B) \mathbf{I},
$$

which indicates that

$$
A \wedge B=0 \Leftrightarrow A \cdot B^{*}=0, \quad A \cdot B=0 \Leftrightarrow A \wedge B^{*}=0 .
$$

Further useful duality relationships are

$$
\begin{aligned}
\mathbf{I}_{\infty O}^{*} & =-\mathbf{I}_{\varepsilon}, & \left(\mathbf{I}_{\infty} \wedge \mathbf{I}_{o}^{\triangleright}\right)^{*}=2 \mathbf{I}_{\varepsilon} \mathbf{e}_{\infty}, \\
\left(\mathbf{I}_{\varepsilon}\left(\mathbf{e}_{o i} \cdot \mathbf{I}_{\infty}\right) \wedge \mathbf{I}_{o}\right)^{*} & =\mathbf{e}_{o i}, & \left(\mathbf{I}_{\varepsilon} \mathbf{I}_{\infty} \wedge\left(\mathbf{e}_{\infty i} \cdot \mathbf{I}_{o}\right)\right)^{*}=-\mathbf{e}_{\infty i}, \\
\left(\mathbf{I}_{\varepsilon}\left(\mathbf{e}_{o} \cdot \mathbf{I}_{\infty}\right) \wedge \mathbf{I}_{o}\right)^{*} & =\mathbf{e}_{o}, & \left(\mathbf{I}_{\varepsilon} \mathbf{I}_{\infty} \wedge\left(\mathbf{e}_{\infty} \cdot \mathbf{I}_{o}\right)\right)^{*}=-\mathbf{e}_{\infty} .
\end{aligned}
$$




\section{CCGA objects}

CCGA is an extension of CGA; thus the objects defined in CGA are also defined in CCGA. The following sections introduce the important definition of a general point in CCGA, and show next how all round and flat geometric objects (point pairs, flat points, circles, lines) of CGA can straightforwardly be embedded in CCGA.

\subsection{Points, point pairs and lines in CCGA}

The point $\mathbf{x}$ of CCGA corresponding to the Euclidean point $\mathbf{x}_{\varepsilon}=x \mathbf{e}_{1}+y \mathbf{e}_{2} \in \mathbb{R}^{2}$ is defined by adding four null vector components ${ }^{4}$

$$
\mathbf{x}=\mathbf{x}_{\varepsilon}+\frac{1}{2}\left(x^{2} \mathbf{e}_{\infty 1}+y^{2} \mathbf{e}_{\infty 2}\right)+x y \mathbf{e}_{\infty 3}+\mathbf{e}_{o} .
$$

Note that the null vector $\mathbf{e}_{o 3}$ is not present in the definition of the point. This is merely to keep the convenient properties of the CGA points, namely, the inner product between two points is identical with the squared distance between them. Let $\mathbf{x}_{1}$ and $\mathbf{x}_{2}$ be two points, their inner product is

$$
\begin{aligned}
\mathbf{x}_{1} \cdot \mathbf{x}_{2}=( & \left(\mathbf{x}_{1 \varepsilon}+\frac{1}{2} x_{1}^{2} \mathbf{e}_{\infty 1}+\frac{1}{2} y_{1}^{2} \mathbf{e}_{\infty 2}+x_{1} y_{1} \mathbf{e}_{\infty 3}+\mathbf{e}_{o}\right) \\
& \cdot\left(\mathbf{x}_{2 \varepsilon}+\frac{1}{2} x_{2}^{2} \mathbf{e}_{\infty 1}+\frac{1}{2} y_{2}^{2} \mathbf{e}_{\infty 2}+x_{2} y_{2} \mathbf{e}_{\infty 3}+\mathbf{e}_{o}\right) .
\end{aligned}
$$

from which together with Table 1 , it follows that

$$
\mathbf{x}_{1} \cdot \mathbf{x}_{2}=\mathbf{x}_{1 \varepsilon} \cdot \mathbf{x}_{2 \varepsilon}-\frac{1}{2}\left(x_{1}^{2}+y_{1}^{2}+x_{2}^{2}+y_{2}^{2}\right) \stackrel{(47)}{=} \mathbf{x}_{1 \mathrm{C}} \cdot \mathbf{x}_{2 \mathrm{C}}=-\frac{1}{2}\left(\mathbf{x}_{1 \varepsilon}-\mathbf{x}_{2 \varepsilon}\right)^{2} .
$$

We see that the inner product is equivalent to minus half of the squared Euclidean distance between $\mathbf{x}_{1}$ and $\mathbf{x}_{2}$.

In the remainder of the paper the following result will be useful, because it relates a point $\mathbf{x}$ in CCGA to the representation $\mathbf{x}_{\mathrm{C}}$ it would have in the subalgebra CGA $\mathbb{R}^{3,1}$ with vector basis $\left\{\mathbf{e}_{o}, \mathbf{e}_{1}, \mathbf{e}_{2}, \mathbf{e}_{\infty}\right\}$.

$$
\begin{aligned}
\mathbf{x} \wedge \mathbf{I}_{\infty}^{\triangleright} & =\left(\mathbf{x}_{\varepsilon}+\frac{1}{2}\left(x^{2} \mathbf{e}_{\infty 1}+y^{2} \mathbf{e}_{\infty 2}\right)+\mathbf{e}_{o}\right) \wedge \mathbf{I}_{\infty}^{\triangleright} \\
& =\left(\mathbf{x}_{\varepsilon}+\mathbf{e}_{o}\right) \wedge \mathbf{I}_{\infty}^{\triangleright}+\frac{1}{2}\left(x^{2} \mathbf{e}_{\infty 1}+y^{2} \mathbf{e}_{\infty 2}\right) \wedge \mathbf{I}_{\infty}^{\triangleright} \\
& \stackrel{(30)}{=}\left(\mathbf{x}_{\varepsilon}+\mathbf{e}_{o}\right) \wedge \mathbf{I}_{\infty}^{\triangleright}+\frac{1}{2}\left(x^{2}+y^{2}\right) \mathbf{e}_{\infty} \wedge \mathbf{I}_{\infty}^{\triangleright} \\
& =\left(\mathbf{x}_{\varepsilon}+\mathbf{e}_{o}\right) \wedge \mathbf{I}_{\infty}^{\triangleright}+\frac{1}{2} \mathbf{x}_{\varepsilon}^{2} \mathbf{e}_{\infty} \wedge \mathbf{I}_{\infty}^{\triangleright} \\
& =\left(\mathbf{x}_{\varepsilon}+\frac{1}{2} \mathbf{x}_{\varepsilon}^{2} \mathbf{e}_{\infty}+\mathbf{e}_{o}\right) \wedge \mathbf{I}_{\infty}^{\triangleright}=\mathbf{x}_{C} \wedge \mathbf{I}_{\infty}^{\triangleright}=\mathbf{x}_{C} \mathbf{I}_{\infty}^{\triangleright},
\end{aligned}
$$

where we have already dropped in the first line the cross term $x y \mathbf{e}_{\infty 3}$, because wedging with $\mathbf{e}_{\infty 3}$, by (11) a factor in $\mathbf{I}_{\infty}^{\triangleright}$, eliminates it. Moreover, the last identity is based on (35). Therefore, if a point in CCGA appears wedged with $\mathbf{I}_{\infty}^{\triangleright}$, we can replace it by the form ${ }^{5}$

$$
\mathbf{x}_{\mathrm{C}}=\mathbf{x}_{\varepsilon}+\frac{1}{2} \mathbf{x}_{\varepsilon}^{2} \mathbf{e}_{\infty}+\mathbf{e}_{o} \stackrel{(18)}{=}-\frac{1}{2}\left(\mathbf{x} \wedge \mathbf{I}_{\infty}^{\triangleright}\right)\left\lfloor\mathbf{I}_{o}^{\triangleright} .\right.
$$

it would have in CGA. This in turn means, that we can embed in CCGA the known CGA representations of round and flat objects, by taking the outer products of between one and four points with $\mathbf{I}_{\infty}^{\triangleright}$, as shown in the following.

\footnotetext{
${ }^{4}$ Note that our definition (43) is different from that given in [10], equation (2).

${ }^{5}$ We have introduced the lower index $C$ for entities in the subalgebra, generated by $\left\{\mathbf{e}_{o}, \mathbf{e}_{1}, \mathbf{e}_{2}, \mathbf{e}_{\infty}\right\}$, isomorphic to $\mathrm{Cl}(3,1)$, the CGA of the two-dimensional Euclidean plane $\mathbb{R}^{2}$.
} 


\subsection{Round and flat objects of CGA in CCGA}

With round objects, we mean points, point pairs and circles with uniform curvature. Similar to CGA, these can be defined by the outer product of one to three points with the simple bivector $\mathbf{I}_{\infty}^{\triangleright}$. Their center $\mathbf{c}_{\mathrm{C}}$, radius $r$ and Euclidean carrier $D$ can be easily extracted. Alternatively, they can be directly constructed from their center $\mathbf{c}_{\mathrm{C}}$, radius $r$ and Euclidean carrier $D$.

Wedging any round object with the point at infinity $\mathbf{e}_{\infty}$, gives the corresponding flat object multivector. From it the orthogonal distance to the origin $\mathbf{c}_{\varepsilon \perp}$ and the Euclidean carrier $D$ can easily be extracted.

We now briefly review the CGA description of round and flat objects embedded in CCGA. The round objects are point, point pair and circle,

$$
\begin{aligned}
P & =\mathbf{x} \wedge \mathbf{I}_{\infty}^{\triangleright}=\mathbf{x}_{\mathrm{C}} \mathbf{I}_{\infty}^{\triangleright}, \\
P p & =\mathbf{x}_{1} \wedge \mathbf{x}_{2} \wedge \mathbf{I}_{\infty}^{\triangleright}=\mathbf{x}_{1 \mathrm{C}} \wedge \mathbf{x}_{2 \mathrm{C}} \mathbf{I}_{\infty}^{\triangleright}, \\
\text { Circle } & =\mathbf{x}_{1} \wedge \mathbf{x}_{2} \wedge \mathbf{x}_{3} \wedge \mathbf{I}_{\infty}^{\triangleright}=\mathbf{x}_{1 \mathrm{C}} \wedge \mathbf{x}_{2 \mathrm{C}} \wedge \mathbf{x}_{3 \mathrm{C}} \mathbf{I}_{\infty}^{\triangleright},
\end{aligned}
$$

The corresponding flat objects are flat point, line and the whole plane,

$$
\begin{aligned}
\text { Flatp } & =P \wedge \mathbf{e}_{\infty}=\mathbf{x} \wedge \mathbf{e}_{\infty} \wedge \mathbf{I}_{\infty}^{\triangleright}=\mathbf{x}_{\mathrm{C}} \wedge \mathbf{e}_{\infty} \mathbf{I}_{\infty}^{\triangleright}, \\
\text { Line } & =P p \wedge \mathbf{e}_{\infty}=\mathbf{x}_{1} \wedge \mathbf{x}_{2} \wedge \mathbf{e}_{\infty} \wedge \mathbf{I}_{\infty}^{\triangleright}=\mathbf{x}_{1 \mathrm{C}} \wedge \mathbf{x}_{2 \mathrm{C}} \wedge \mathbf{e}_{\infty} \mathbf{I}_{\infty}^{\triangleright}, \\
\text { Plane } & =\text { Circle } \wedge \mathbf{e}_{\infty}=\mathbf{x}_{1} \wedge \mathbf{x}_{2} \wedge \mathbf{x}_{3} \wedge \mathbf{e}_{\infty} \wedge \mathbf{I}_{\infty}^{\triangleright}=\mathbf{x}_{1 \mathrm{C}} \wedge \mathbf{x}_{2 \mathrm{C}} \wedge \mathbf{x}_{3 \mathrm{C}} \wedge \mathbf{e}_{\infty} \mathbf{I}_{\infty}^{\triangleright} .
\end{aligned}
$$

The above embeddings by means of the outer product with $\mathbf{I}_{\infty}^{\triangleright}$, allow to make use of standard CGA results found in [9]. All embedded round entities of point, point pair and circle have one common multivector form ${ }^{6}$

$$
\begin{aligned}
S & =\left(D \wedge \mathbf{c}_{\varepsilon}+\left[\frac{1}{2}\left(\mathbf{c}_{\varepsilon}^{2}+r^{2}\right) D-\mathbf{c}_{\varepsilon} \mathbf{c}_{\varepsilon}\right\rfloor D\right] \mathbf{e}_{\infty}+D \mathbf{e}_{o}+D\left\lfloor\mathbf{c}_{\varepsilon} E\right) \mathbf{I}_{\infty}^{\triangleright}=S_{\mathrm{C}} \mathbf{I}_{\infty}^{\triangleright}, \\
S_{\mathrm{C}} & =-\frac{1}{2} S\left\lfloor\mathbf{I}_{o}^{\triangleright} .\right.
\end{aligned}
$$

The Euclidean carriers $D$ are for each object

$$
D=\left\{\begin{aligned}
1, & \text { point } \mathbf{x} \\
\mathbf{d}_{\varepsilon}, & \text { point pair } P p \\
\mathbf{I}_{\varepsilon}, & \text { circle Circle },
\end{aligned}\right.
$$

where the unit point pair connection direction vector is $\mathbf{d}_{\varepsilon}=\left(\mathbf{x}_{1 \varepsilon}-\mathbf{x}_{2 \varepsilon}\right) / 2 r$. The radius $r$ of a round object and its center $\mathbf{c}_{\mathrm{C}}$ are generally determined by

$$
r^{2}=\frac{S_{\mathrm{C}} \widetilde{S_{\mathrm{C}}}}{\left(S_{\mathrm{C}} \wedge \mathbf{e}_{\infty}\right)\left(S_{\mathrm{C}} \wedge \mathbf{e}_{\infty}\right) \sim}, \quad \mathbf{c}_{\mathrm{C}}=S_{\mathrm{C}} \mathbf{e}_{\infty} S_{\mathrm{C}} .
$$

where $\widetilde{S_{\mathrm{C}}}$ indicates the reverse of $S_{\mathrm{C}}$. By further wedging a circle $S$ with the simple bivector $\mathbf{I}_{O}^{\triangleright}$ we obtain a 7-vector and its dual

$$
S \wedge \mathbf{I}_{o}^{\triangleright}=\mathbf{x}_{1} \wedge \mathbf{x}_{2} \wedge \mathbf{x}_{3} \wedge \mathbf{I}_{\infty}^{\triangleright} \wedge \mathbf{I}_{o}^{\triangleright}=\mathbf{x}_{1 \mathrm{C}} \wedge \mathbf{x}_{2 \mathrm{C}} \wedge \mathbf{x}_{3 \mathrm{C}} \mathbf{I}^{\triangleright}=\left(\mathbf{c}_{\mathrm{C}}-\frac{1}{2} r^{2} \mathbf{e}_{\infty}\right) \mathbf{I}
$$

\footnotetext{
${ }^{6}$ Note, that the left- and right contraction, respectively, are needed essentially. Albeit, because the two are related by reversion, $A\rfloor B=\widetilde{B} \mid \widetilde{A}$, and reversion only changes signs of blades, it would be possible to use only one of the two forms of the contraction, and express the other by reordering and sign changes.
} 
where the dual vector $\mathbf{c}_{\mathrm{C}}-\frac{1}{2} r^{2} \mathbf{e}_{\infty}$ is exactly the dual vector of the sphere $S_{\mathrm{C}}$ in CGA

$$
S_{\mathrm{C}}^{*}=\mathbf{c}_{\mathrm{C}}-\frac{1}{2} r^{2} \mathbf{e}_{\infty}=S_{\mathrm{C}} \mathbf{I}_{\mathrm{C}}^{-1} \text {. }
$$

All embedded flat entities of flat point, line and plane have one common multivector form

$$
\begin{aligned}
F & =S \wedge \mathbf{e}_{\infty}=\left(D \wedge \mathbf{c}_{\varepsilon} \mathbf{e}_{\infty}-D E\right) \mathbf{I}_{\infty}^{\triangleright}=\left(D \mathbf{c}_{\varepsilon \perp} \mathbf{e}_{\infty}-D E\right) \mathbf{I}_{\infty}^{\triangleright}=F_{\mathrm{C}} \mathbf{I}_{\infty}^{\triangleright}, \\
F_{\mathrm{C}} & =S_{\mathrm{C}} \wedge \mathbf{e}_{\infty}=-\frac{1}{2} F\left\lfloor\mathbf{I}_{o}^{\triangleright} .\right.
\end{aligned}
$$

where the orthogonal Euclidean distance of the flat object from the origin is

$$
\mathbf{c}_{\varepsilon \perp}=\left\{\begin{aligned}
\mathbf{x}_{\varepsilon}, & \text { finite-infinite point pair Flatp } \\
\mathbf{c}_{\varepsilon \perp}, & \text { line Line } \\
0, & \text { plane Plane },
\end{aligned}\right.
$$

The Euclidean carrier blade $D$, and the orthogonal Euclidean distance vector of $F$ from the origin, can both be directly determined from the flat object multivector as

$$
D=-F_{\mathrm{C}}\left\lfloor E, \quad \mathbf{c}_{\varepsilon \perp}=D^{-1}\left(F_{\mathrm{C}} \wedge \mathbf{e}_{o}\right)\lfloor E .\right.
$$

By further wedging a line $F$ with the simple bivector $\mathbf{I}_{o}^{\triangleright}$ we obtain a 7-vector and its dual

$$
F \wedge \mathbf{I}_{o}^{\triangleright}=\mathbf{x}_{1} \wedge \mathbf{x}_{2} \wedge \mathbf{e}_{\infty} \wedge \mathbf{I}_{\infty}^{\triangleright} \wedge \mathbf{I}_{o}^{\triangleright}=\mathbf{x}_{1 \mathrm{C}} \wedge \mathbf{x}_{2 C} \wedge \mathbf{e}_{\infty} \mathbf{I}^{\triangleright}=2\left|\mathbf{x}_{2 \varepsilon}-\mathbf{x}_{1 \varepsilon}\right|\left(\mathbf{n}_{\varepsilon}+d \mathbf{e}_{\infty}\right) \mathbf{I},
$$

where up to the factor $2\left|\mathbf{x}_{2 \varepsilon}-\mathbf{x}_{1 \varepsilon}\right|$ the dual vector $\mathbf{n}_{\varepsilon}+d \mathbf{e}_{\infty}$ is exactly the dual vector of the line $F_{\mathrm{C}}$ in CGA

$$
F_{\mathrm{C}}^{*}=F_{\mathrm{C}} \mathbf{I}_{\mathrm{C}}^{-1}=\left|\mathbf{x}_{2 \varepsilon}-\mathbf{x}_{1 \varepsilon}\right|\left(\mathbf{n}_{\varepsilon}+d \mathbf{e}_{\infty}\right) \propto \mathbf{n}_{\varepsilon}+d \mathbf{e}_{\infty},
$$

with Euclidean unit normal vector to the line $\mathbf{n}_{\varepsilon}=-\left(\mathbf{x}_{2 \varepsilon}-\mathbf{x}_{1 \varepsilon}\right) \mathbf{I}_{\varepsilon} /\left|\mathbf{x}_{2 \varepsilon}-\mathbf{x}_{1 \varepsilon}\right|$ and shortest distance of the line $F$ from the origin $d=-\left(\mathbf{x}_{1 \varepsilon} \wedge \mathbf{x}_{2 \varepsilon}\right) \mathbf{I}_{\varepsilon} /\left|\mathbf{x}_{2 \varepsilon}-\mathbf{x}_{1 \varepsilon}\right|$.

Because by (45) the distance between two points is given by their inner product, the vector $F_{\mathrm{C}}^{*}$ like in CGA can also be found as the difference of two points $\mathbf{y}_{1}$ and $\mathbf{y}_{2}$, bisected by the same line $F$, i.e. $F_{\mathrm{C}}^{*} \propto \mathbf{y}_{2}-\mathbf{y}_{1}$.

\section{Conics}

This section describes how CCGA handles the conics. All embedded CGA objects in CCGA defined in Section 3 are thus part of a more general framework.

\subsection{General conics in two Euclidean dimensions}

A conic in $\mathbb{R}^{2}$ is formulated as

$$
F(x, y)=\mathrm{a} x^{2}+\mathrm{b} y^{2}+\mathrm{c} x y+\mathrm{d} x+\mathrm{e} y+\mathrm{f}=0 .
$$

A conic is constructed by wedging five points together as follows

$$
\mathbf{q}=\mathbf{x}_{1} \wedge \mathbf{x}_{2} \wedge \mathbf{x}_{3} \wedge \mathbf{x}_{4} \wedge \mathbf{x}_{5}
$$

The multivector blade $\mathbf{q}$ corresponds to the primal form of a conic in CCGA, with grade 5 and 6 components specified by the six coefficients $a, b, \ldots$, f. If we further 
wedge the 5-blade $\mathbf{q}$ with the simple bivector $\mathbf{I}_{o}^{\triangleright}$ we obtain a 7-blade and its dual vector $\left(\mathbf{q} \wedge \mathbf{I}_{o}^{\triangleright}\right)^{*}$ as

$$
\begin{aligned}
\mathbf{q} \wedge \mathbf{I}_{o}^{\triangleright}= & -\mathbf{I}_{\varepsilon}\left(\left(2 \mathrm{a} \mathbf{e}_{o 1}+2 \mathrm{~b} \mathbf{e}_{o 2}+\mathrm{c} \mathbf{e}_{o 3}\right) \cdot \mathbf{I}_{\infty}\right) \wedge \mathbf{I}_{o}+\left(\mathrm{d} \mathbf{e}_{1}+\mathrm{e} \mathbf{e}_{2}\right) \mathbf{I}_{\varepsilon} \mathbf{I}_{\infty o} \\
& +\mathrm{f} \mathbf{I}_{\varepsilon} \mathbf{I}_{\infty} \wedge\left(\mathbf{e}_{\infty} \cdot \mathbf{I}_{o}\right) \\
= & \left(-\left(2 \mathrm{a} \mathbf{e}_{o 1}+2 \mathrm{~b} \mathbf{e}_{o 2}+\mathrm{c} \mathbf{e}_{o 3}\right)+\mathrm{d} \mathbf{e}_{1}+\mathrm{e} \mathbf{e}_{2}-\mathrm{f} \mathbf{e}_{\infty}\right) \mathbf{I}=\left(\mathbf{q} \wedge \mathbf{I}_{o}^{\triangleright}\right)^{*} \mathbf{I},
\end{aligned}
$$

where in the second equality we used the duality relationships of (42). The expression for the dual vector $\left(\mathbf{q} \wedge \mathbf{I}_{o}^{\triangleright}\right)^{*}$ is therefore

$$
\left(\mathbf{q} \wedge \mathbf{I}_{o}^{\triangleright}\right)^{*}=-\left(2 \mathrm{a} \mathbf{e}_{o 1}+2 \mathrm{~b} \mathbf{e}_{o 2}+\mathrm{c} \mathbf{e}_{o 3}\right)+\mathrm{d} \mathbf{e}_{1}+\mathrm{e} \mathbf{e}_{2}-\mathrm{f} \mathbf{e}_{\infty} .
$$

Proposition 4.1. A point $\mathbf{x}$ lies on the conic curve defined by five points, i.e. by their outer product $\mathbf{q}$, if and only if $\mathbf{x} \wedge\left(\mathbf{q} \wedge \mathbf{I}_{o}^{\triangleright}\right)=0$.

Proof.

$$
\begin{aligned}
\mathbf{x} \wedge\left(\mathbf{q} \wedge \mathbf{I}_{o}^{\triangleright}\right) & =\mathbf{x} \wedge\left(\left(\mathbf{q} \wedge \mathbf{I}_{o}^{\triangleright}\right)^{*} \mathbf{I}\right)=\mathbf{x} \cdot\left(\mathbf{q} \wedge \mathbf{I}_{o}^{\triangleright}\right)^{*} \mathbf{I} \\
& =\mathbf{x} \cdot\left(-\left(2 \mathrm{a} \mathbf{e}_{o 1}+2 \mathrm{~b} \mathbf{e}_{o 2}+\mathrm{c} \mathbf{e}_{o 3}\right)+\left(\mathrm{d} \mathbf{e}_{1}+\mathrm{e} \mathbf{e}_{2}\right)-\mathrm{f} \mathbf{e}_{\infty}\right) \mathbf{I} \\
& =\left(\mathrm{a} x^{2}+\mathrm{b} y^{2}+\mathrm{c} x y+\mathrm{d} x+\mathrm{e} y+\mathrm{f}\right) \mathbf{I} .
\end{aligned}
$$

This indeed corresponds to the formula (64) representing a general conic.

Proposition 4.1 together with (67) yields the inner product condition for a point $\mathbf{x}$ to be on the conic, expressed by

Corollary 4.2. A point $\mathbf{x}$ lies on the conic defined by $\mathbf{q}$, if and only if $\mathbf{x} \cdot\left(\mathbf{q} \wedge \mathbf{I}_{o}^{\triangleright}\right)^{*}=0$.

The six coefficients $\{a, \ldots, f\}$ of the conic equation (64) can be easily extracted from the conic 5-blade $\mathbf{q}$ of (65) by computing the following scalar products with vector $\left(\mathbf{q} \wedge \mathbf{I}_{o}^{\triangleright}\right)^{*}$ as

$$
\begin{aligned}
& a=\frac{1}{2}\left(\mathbf{q} \wedge \mathbf{I}_{o}^{\triangleright}\right)^{*} \cdot \mathbf{e}_{\infty 1}, \quad b=\frac{1}{2}\left(\mathbf{q} \wedge \mathbf{I}_{o}^{\triangleright}\right)^{*} \cdot \mathbf{e}_{\infty 2}, \quad c=\left(\mathbf{q} \wedge \mathbf{I}_{o}^{\triangleright}\right)^{*} \cdot \mathbf{e}_{\infty 3}, \\
& d=\left(\mathbf{q} \wedge \mathbf{I}_{o}^{\triangleright}\right)^{*} \cdot \mathbf{e}_{1}, \quad e=\left(\mathbf{q} \wedge \mathbf{I}_{o}^{\triangleright}\right)^{*} \cdot \mathbf{e}_{2}, \quad f=\left(\mathbf{q} \wedge \mathbf{I}_{o}^{\triangleright}\right)^{*} \cdot \mathbf{e}_{o} .
\end{aligned}
$$

\subsection{Axis aligned conics}

By wedging a point $\mathbf{x}$ with $\mathbf{e}_{\infty 3}$, the mixed coordinate term $x y \mathbf{e}_{\infty 3}$ in (43) is removed. This means, that an axis aligned conic can be constructed from the outer product of any four distinct points $\mathbf{x}_{1}, \mathbf{x}_{2}, \mathbf{x}_{3}, \mathbf{x}_{4}$ on the conic in the following way

$$
\mathbf{q}=\mathbf{x}_{1} \wedge \mathbf{x}_{2} \wedge \mathbf{x}_{3} \wedge \mathbf{x}_{4} \wedge \mathbf{e}_{\infty 3} .
$$

The dual vector of the 7-vector $\mathbf{q} \wedge \mathbf{I}_{o}^{\triangleright}$ is

$$
\left(\mathbf{q} \wedge \mathbf{I}_{o}^{\triangleright}\right)^{*}=-\left(2 \mathrm{a} \mathbf{e}_{o 1}+2 \mathrm{~b} \mathbf{e}_{o 2}\right)+\mathrm{d} \mathbf{e}_{1}+\mathrm{ee}_{2}-\mathrm{f} \mathbf{e}_{\infty} .
$$

A general point $\mathbf{x}$ is on the conic represented by the 5-blade $\mathbf{q}$ in (70), if and only if,

$$
\begin{aligned}
\mathbf{x} \wedge\left(\mathbf{q} \wedge \mathbf{I}_{o}^{\triangleright}\right) & =\mathbf{x} \wedge\left(\left(\mathbf{q} \wedge \mathbf{I}_{o}^{\triangleright}\right)^{*} \mathbf{I}\right)=\mathbf{x} \cdot\left(\mathbf{q} \wedge \mathbf{I}_{o}^{\triangleright}\right)^{*} \mathbf{I} \\
& =\mathbf{x} \cdot\left(-\left(2 \mathrm{ae}_{o 1}+2 \mathrm{~b} \mathbf{e}_{o 2}\right)+\left(\mathrm{d} \mathbf{e}_{1}+\mathrm{e} \mathbf{e}_{2}\right)-\mathrm{f} \mathbf{e}_{\infty}\right) \mathbf{I} \\
& =\left(\mathrm{a} x^{2}+\mathrm{b} y^{2}+\mathrm{d} x+\mathrm{e} y+\mathrm{f}\right) \mathbf{I} .
\end{aligned}
$$




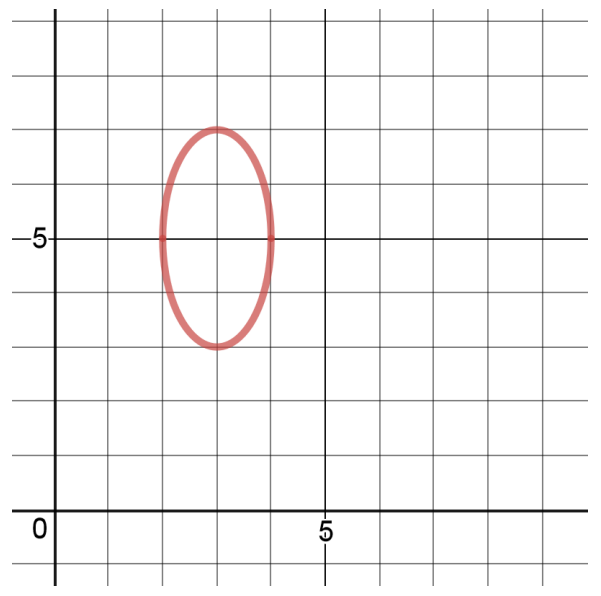

FIGURE 1. An axis aligned ellipse with half axis 1 and 2, and center coordinates $\left(c_{x}=3, c_{y}=5\right)$. Graph with [3].

which in turn means that we get from $\mathbf{x} \wedge\left(\mathbf{q} \wedge \mathbf{I}_{o}^{\triangleright}\right)=0$, or equivalently from $\mathbf{x} \cdot(\mathbf{q} \wedge$ $\left.\mathbf{I}_{o}^{\triangleright}\right)^{*}=0$, the implicit equation for an axis aligned conic

$$
a x^{2}+b x^{2}+d x+e y+f=0 .
$$

We note that for the representation of axis aligned conics the dimensions $\mathbf{e}_{o 3}$ and $\mathbf{e}_{\infty 3}$ are superfluous, and axis aligned conics could therefore be represented in the lower dimensional geometric algebra $C l(4,2)$, which is a version for two Euclidean dimensions of the model for axis aligned quadrics in the geometric algebra $\mathrm{Cl}(6,3)$ previously proposed for three Euclidean dimensions in [15]. But by keeping the higher dimensional embedding of axis aligned conics in $C l(5,3)$, we preserve the freedom of performing arbitrary rotations as explained in Section 6, which is not possible in $C l(4,2)$.

For example, if we choose the four points $\mathbf{x}_{1}, \mathbf{x}_{2}, \mathbf{x}_{3}, \mathbf{x}_{4}$ with coordinates

$$
x_{1}=4, y_{1}=5, \quad x_{2}=3, y_{2}=7, \quad x_{3}=2, y_{3}=5, \quad x_{4}=3, y_{4}=3,
$$

of an axis aligned ellipse with half axis 1 and 2, and center coordinates $\left(c_{x}=3, c_{y}=\right.$ 5), we can use e.g. the Clifford Multivector Toolbox (for MATLAB) [14] to obtain from $\mathbf{q}$ the ellipse parameters by applying (69) as

$$
a=-16, \quad b=-4, \quad d=96, \quad e=40, \quad f=-228,
$$

where as expected $c=\left(\mathbf{q} \wedge \mathbf{I}_{o}^{\triangleright}\right)^{*} \cdot \mathbf{e}_{\infty 3}=0$, for an axis aligned ellipse. Figure 1 shows the resulting ellipse.

Note, that the circle (50) is a special case of an axis aligned conic, because

$$
\text { Circle }=\mathbf{x}_{1} \wedge \mathbf{x}_{2} \wedge \mathbf{x}_{3} \wedge \mathbf{I}_{\infty}^{\triangleright}=\mathbf{x}_{1} \wedge \mathbf{x}_{2} \wedge \mathbf{x}_{3} \wedge \mathbf{e}_{\infty 12}^{\triangleright} \wedge \mathbf{e}_{\infty 3},
$$

obtained from (70) by replacing $\mathbf{x}_{4}$ with the vector $\mathbf{e}_{\infty 12}^{\triangleright}$. 


\section{Intersections}

Any number of linearly independent round or flat embedded CGA objects in CCGA and any number of conics $\{\mathbf{A}, \mathbf{B}, \ldots, \mathbf{Z}\}$, after wedging with the simple bivector $\mathbf{I}_{o}^{\triangleright}$, can be intersected by computing the dual of the outer product of their duals

$$
\left(\text { intersect } \wedge \mathbf{I}_{o}^{\triangleright}\right)^{*}=\left(\mathbf{A} \wedge \mathbf{I}_{o}^{\triangleright}\right)^{*} \wedge\left(\mathbf{B} \wedge \mathbf{I}_{o}^{\triangleright}\right)^{*} \wedge \ldots \wedge\left(\mathbf{Z} \wedge \mathbf{I}_{o}^{\triangleright}\right)^{*} .
$$

The criterion for a general point $\mathbf{x}$ to be on the intersection is

$$
\mathbf{x} \cdot\left(\text { intersect } \wedge \mathbf{I}_{o}^{\triangleright}\right)^{*}=0 .
$$

For cases that one object is wholly included in another object (like a line in a plane), the proper meet operation has to be defined, taking into account the subspace spanned by the join of the two objects [11].

As an example, we intersect the two conics $\mathbf{q}_{1}$ of (65) and its axis aligned version $\mathbf{q}_{2}$ of (70) (formed from the same first four points) yielding the quadvector

$$
\text { intersect }_{4}=-\frac{1}{2}\left(\left(\left(\mathbf{q}_{1} \wedge \mathbf{I}_{o}^{\triangleright}\right)^{*} \wedge\left(\mathbf{q}_{2} \wedge \mathbf{I}_{o}^{\triangleright}\right)^{*}\right) \mathbf{I}\right)\left\lfloor\mathbf{I}_{\infty}^{\triangleright} \propto \mathbf{x}_{1} \wedge \mathbf{x}_{2} \wedge \mathbf{x}_{3} \wedge \mathbf{x}_{4},\right.
$$

that is exactly proportional to the outer product of the four intersection points.

\section{Versors for rotation, translation and scaling}

We acknowledge inspiration from the related formalism in [10]. Note that the conic conformal point definition (2) of [10] differs from our definition (43), which partly accounts for the differences and simplifications in the versor expressions for rotations and especially translations below.

For the successful implementation of rotations together with a simplification of the translation versors, we found it essential to define the null vector pair $\left\{\mathbf{e}_{\infty 3}, \mathbf{e}_{o 3}\right\}$ in the symmetric fashion of (2)

$$
\mathbf{e}_{\infty 3}=\frac{1}{\sqrt{2}}\left(\mathbf{e}_{+3}+\mathbf{e}_{-3}\right), \quad \mathbf{e}_{o 3}=\frac{1}{\sqrt{2}}\left(\mathbf{e}_{-3}-\mathbf{e}_{+3}\right) .
$$

Only with this definition ${ }^{7}$ for $\left\{\mathbf{e}_{\infty 3}, \mathbf{e}_{o 3}\right\}$, were we able to reduce the number of versor factors for achieving translations from the six needed in [10], to the four featured below in (89). Moreover, as seen below, our approach has the additional advantage of completely eliminating the quadratic terms in the translation vector coordinates from the exponents of the factors in the translation operators, compared to [10].

Rotations are generated by the following three bivectors

$$
e_{12}, \quad B_{2}=\frac{1}{2} \mathbf{e}_{o 3} \mathbf{e}_{\infty 12}^{\triangleright}, \quad B_{3}=\mathbf{e}_{\infty 3} \mathbf{e}_{o 12}^{\triangleright}, \quad B_{2}^{2}=B_{3}^{2}=0,
$$

in the rotor form of $R=R_{1}\left(R_{2} \wedge R_{3}\right)$, where

$$
R_{1}=e^{\frac{\varphi}{2} e_{12}}, \quad R_{2}=\cos \varphi+\sin \varphi B_{2}, \quad R_{3}=\cos \varphi+\sin \varphi B_{3} .
$$

\footnotetext{
${ }^{7}$ For $\left\{\mathbf{e}_{\infty 1}, \mathbf{e}_{o 1}\right\}$ and $\left\{\mathbf{e}_{\infty 2}, \mathbf{e}_{o 2}\right\}$, we could also have chosen $\lambda_{1}=\lambda_{2}=\sqrt{2}$ as in [10], without altering our form of the transformation versors given below. But for aesthetic reasons, we decided in (2) to simply set all three $\lambda$ coefficients to 1 . For ease of numerical implementation, the choice $\lambda_{1}=\lambda_{2}=\sqrt{2}$ might be of advantage, but $\lambda_{3}=1$ has to be preserved if the current versor formulation is to be adopted.
} 
Applying the rotor $R$ to a conic conformal point $\mathbf{x}$ leads to

$$
\mathbf{x}^{\prime}=\widetilde{R} \mathbf{x} R, \mathbf{x}_{\varepsilon}^{\prime}=x^{\prime} \mathbf{e}_{1}+y^{\prime} \mathbf{e}_{2}, x^{\prime}=x \cos \varphi-y \sin \varphi, y^{\prime}=x \sin \varphi+y \cos \varphi,
$$

an anticlockwise (mathematically positive) rotation in the $\mathbf{I}_{\mathcal{\varepsilon}}$-plane. Our formulation of the rotors above has some differences to Proposition 5.1 in [10], but is of the same level of algebraic complexity.

We note the useful invariance relationships, that

$$
\widetilde{R} \mathbf{a} R=\mathbf{a} \quad \forall \mathbf{a} \in\left\{1, \mathbf{e}_{o}, \mathbf{e}_{\infty}, E, \mathbf{I}, \mathbf{I}_{\varepsilon}, \mathbf{I}_{\mathrm{C}}, \mathbf{I}_{\infty o}, \mathbf{I}_{\infty}, \mathbf{I}_{o}, \mathbf{I}_{\infty}^{\triangleright}, \mathbf{I}_{o}^{\triangleright}\right\} .
$$

Because embedded flat and round CGA objects and all conics are constructed from outer products of between one and five points and $\mathbf{I}_{\infty}^{\triangleright}$, these objects are naturally covariant under rotations. Another consequence of this invariance is, that equations like (77), involving outer products with $\mathbf{I}_{o}^{\triangleright}$, are also covariant under rotations. Rotation covariance will certainly be of great value, when CCGA is employed, e.g., for the construction of feature multivectors. We further note, that in general the vector $\mathbf{e}_{\infty 3}$ is not rotation invariant, which is natural for an outer product factor used to yield axis aligned conics, which being axis aligned, can not be expected to be rotation covariant.

Different from [10], translation by distance $a \in \mathbb{R}$ in the direction of $\mathbf{e}_{1}$ is achieved by the versor $T_{x}=T_{1} T_{2}=T_{2} T_{1}$, with

$$
T_{1}=e^{\frac{1}{2} a \mathbf{e}_{1} \mathbf{e}_{\infty 1}}=1+\frac{1}{2} a \mathbf{e}_{1} \mathbf{e}_{\infty 1}, \quad T_{2}=e^{\frac{1}{2} a \mathbf{e}_{2} \mathbf{e}_{\infty 3}}=1+\frac{1}{2} a \mathbf{e}_{2} \mathbf{e}_{\infty 3},
$$

which leads to

$$
\mathbf{x}^{\prime}=\widetilde{T}_{x} \mathbf{x} T_{x}, \quad \mathbf{x}_{\mathcal{\varepsilon}}^{\prime}=(x+a) \mathbf{e}_{1}+y \mathbf{e}_{2} .
$$

Note, that the product of the two null bivector generators $\left(\mathbf{e}_{1} \mathbf{e}_{\infty 1}\right)\left(\mathbf{e}_{2} \mathbf{e}_{\infty 3}\right)$, commutes and is a null quadvector (squaring to zero).

Translation by distance $b \in \mathbb{R}$ in the direction of $\mathbf{e}_{2}$ is similarly achieved by the versor $T_{y}=T_{3} T_{4}=T_{4} T_{3}$, with

$$
T_{3}=e^{\frac{1}{2} b \mathbf{e}_{2} \mathbf{e}_{\infty 2}}=1+\frac{1}{2} b \mathbf{e}_{2} \mathbf{e}_{\infty 2}, \quad T_{4}=e^{\frac{1}{2} b \mathbf{e}_{1} \mathbf{e}_{\infty 3}}=1+\frac{1}{2} b \mathbf{e}_{1} \mathbf{e}_{\infty 3},
$$

which leads to

$$
\mathbf{x}^{\prime}=\widetilde{T}_{y} \mathbf{x} T_{y}, \quad \mathbf{x}_{\varepsilon}^{\prime}=x \mathbf{e}_{1}+(y+b) \mathbf{e}_{2} .
$$

We note the commutation relations

$$
T_{1} T_{3}=T_{3} T_{1}, \quad T_{2} T_{4}=T_{4} T_{2}, \quad \text { but } \quad T_{x} T_{y} \neq T_{y} T_{x} .
$$

For example, $T_{1}$ and $T_{3}$ commute, because the disjoint bivector generators $\mathbf{e}_{1} \mathbf{e}_{\infty 1}$ and $\mathbf{e}_{2} \mathbf{e}_{\infty 2}$ obviously commute. But $T_{1}$ and $T_{4}$ do not commute, because the bivector generators multiply to $\mathbf{e}_{1} \mathbf{e}_{\infty 1} \mathbf{e}_{1} \mathbf{e}_{\infty 3}=-\mathbf{e}_{1} \mathbf{e}_{\infty 3} \mathbf{e}_{1} \mathbf{e}_{\infty 1}$.

In total we have for the application to conic conformal points

$$
\mathbf{x}^{\prime}=\widetilde{T} \mathbf{x} T, \quad T=T_{x} T_{y}=T_{1} T_{2} T_{3} T_{4}, \quad \mathbf{x}_{\varepsilon}^{\prime}=(x+a) \mathbf{e}_{1}+(y+b) \mathbf{e}_{2} .
$$

It is interesting to note, that in spite of (88), we do have applied to any point $\mathbf{x}$ that

$$
\mathbf{x}^{\prime}=\widetilde{T} \mathbf{x} T=\widetilde{T_{x} T_{y}} \mathbf{x} T_{x} T_{y}=\widetilde{T_{y} T_{x}} \mathbf{x} T_{y} T_{x} .
$$

This commutation means, that one can first apply $T_{x}$, obtain the point at position $(x+a) \mathbf{e}_{1}+y \mathbf{e}_{2}$, and then apply $T_{y}$ to obtain the point at position $(x+a) \mathbf{e}_{1}+(y+$ 
b) $\mathbf{e}_{2}$, or in reverse order to obtain the same shift in both $x$ and $y$ directions. The versor operators themselves do not commute (88), but applied to the point at position $x \mathbf{e}_{1}+y \mathbf{e}_{2}$ they do.

We further note, that Proposition 5.2 [10] needs six factors for a general translation, whereas we only need four factors. Equations (25) and (28) in Proposition 5.2 [10] are versors with quadratic terms in displacement distances $a, b$, which we do not need in (84) and (86). We have only bivector terms linear in displacement distances $a, b$, in each of the four versors $T_{1}, \ldots T_{4}$. We expect this to be advantageous in application to optimization problems.

We note the useful invariance relationships, that

$$
\widetilde{T} \mathbf{a} T=\mathbf{a} \quad \forall \mathbf{a} \in\left\{1, \mathbf{e}_{\infty 1}, \mathbf{e}_{\infty 2}, \mathbf{e}_{\infty 3}, \mathbf{e}_{\infty}, \mathbf{I}, \mathbf{I}_{\infty}, \mathbf{I}_{\infty}^{\triangleright}\right\} .
$$

By construction therefore all flat and round CGA objects and all conics are translation covariant, this includes, because of $T \mathbf{e}_{\infty 3} \widetilde{T}=\mathbf{e}_{\infty 3}$, axis aligned conics as well, since translation has no effect on axis alignment.

As in [10], scaling by positive scalar $\alpha \in \mathbb{R}$ is achieved with the help of the scaling operator (scalor) $S=S_{1} S_{2} S_{3}$, where

$$
S_{k}=\frac{1}{2}\left(\frac{\alpha+1}{\sqrt{\alpha}}+\frac{\alpha-1}{\sqrt{\alpha}} E_{k}\right), \quad \widetilde{S_{k}} S_{k}=S_{k} \widetilde{S_{k}}=1, \quad 1 \leq k \leq 3 .
$$

Note that the three factors $S_{k}, 1 \leq k \leq 3$, mutually commute. This leads to (isotropic) scaling of points

$$
\mathbf{x}^{\prime}=\alpha \widetilde{S} \mathbf{x} S, \quad \mathbf{x}_{\varepsilon}^{\prime}=\alpha \mathbf{x}_{\varepsilon} .
$$

Note that the overall factor $\alpha$ could be omitted in $\mathbf{x}^{\prime}=\alpha S \mathbf{x} \widetilde{S}$, due to the homogeneity of the conic point representation $\mathbf{x}$, but we include it for convenience, such that $\mathbf{x}^{\prime} \cdot \mathbf{e}_{\infty}=\mathbf{x} \cdot \mathbf{e}_{\infty}=-1$.

The following multivector elements are invariant under scaling (93)

$$
\mathbf{e}_{o}, \mathbf{e}_{o 1}, \mathbf{e}_{o 2}, \mathbf{e}_{o 3}, \mathbf{I}_{o}, \mathbf{I}_{o 12}, \mathbf{I}_{o}^{\triangleright}, \mathbf{e}_{o 12}^{\triangleright} .
$$

For bivectors this means

$$
\left\{\mathbf{I}_{o 12}, \mathbf{I}_{o}^{\triangleright}\right\}=\alpha^{2} \widetilde{S}\left\{\mathbf{I}_{o 12}, \mathbf{I}_{o}^{\triangleright}\right\} S,
$$

and for the trivector

$$
\mathbf{I}_{o}=\alpha^{3} \widetilde{S} \mathbf{I}_{o} S
$$

Because conics (65) are constructed from outer products of points, the scaling operators $S_{k}, k=1,2,3$, of (92) act (even individually) via outermorphisms covariantly on conics. And the expressions for intersections of conics (77), also remain covariant under scaling (93), because scaling (93) maps $\mathbf{I} \rightarrow \alpha^{8} \mathbf{I}$ to a scalar multiple of itself, and because the representation of points and conics is homogeneous.

Furthermore, due to the relationships (8) and (9) one can show that the scalor (93) maps the three infinity vectors $\left\{\mathbf{e}_{\infty 1}, \mathbf{e}_{\infty 2}, \mathbf{e}_{\infty 3}\right\} \rightarrow \alpha^{2}\left\{\mathbf{e}_{\infty 1}, \mathbf{e}_{\infty 2}, \mathbf{e}_{\infty 3}\right\}$ to scalar multiples of themselves. This together with (94), that means that scaling can be covariantly applied to axis aligned conics, due to the homogeneity of the CCGA representation. This also applies to intersection operations involving axis aligned conics. 
Isotropic scaling does apply to embedded CGA objects, because it simply $\operatorname{maps} \mathbf{e}_{\infty} \rightarrow \alpha^{2} \mathbf{e}_{\infty}, \mathbf{I}_{\infty}^{\triangleright} \rightarrow \alpha^{4} \mathbf{I}_{\infty}^{\triangleright}, \mathbf{I}_{\infty 12} \rightarrow \alpha^{4} \mathbf{I}_{\infty 12}$, and $\mathbf{I}_{\infty} \rightarrow \alpha^{6} \mathbf{I}_{\infty}$, to scalar multiples of themselves, respectively, which is no problem due to the homogeneity of the representation.

Translators permit rotations and scaling relative to arbitrary centers of rotation and scaling, by first translating the respective center position to the origin, rotating or scaling with the above versors $R, S$, followed by back translation to the center.

Alternatively, it is possible to combine conic conformal geometric algebra with double conformal geometric algebra (DCGA) [6]. For that the coefficients of the conic $a, b, c, d, e, f$, can be extracted with (69) from q (see (75) for an example), and then used to define the same conic in DCGA. The rotors for reflection, rotation, translation and scaling are substantially simpler in DCGA. So if preferred, versor operations can be executed within DCGA, and the coefficients $a^{\prime}, b^{\prime}, c^{\prime}, d^{\prime}, e^{\prime}, f^{\prime}$, of the resulting new conic can be extracted from its DCGA bivector representation and transferred back to CCGA to define the same conic in CCGA.

\section{Conclusion}

This work on conic CGA (CCGA) $C l(5,3)$ provides a detailed algebraic study and refinement of C. Perwass' ansatz [13]. An alternative recent approach we refer to is by J. Hrdina et al [10]. The complete CGA of two-dimensional Euclidean geometry appears as algebraic subset of CCGA. We showed how in CCGA conics can be defined from outer products of five on-curve points. This enables the computation of general intersections by outer products of duals of conics. We showed that CCGA has versor operations, and compared with the versors given in [10]. A modification of basic algebraic definitions allowed us to simplify the formulation of translators to the form they had in [13], while preserving the full consistent set of versors for the operations of rotation, translation and scaling.

Nevertheless, it can be said, that versors in double CGA (DCGA) [1] are structurally simpler and a hybrid approach where the corresponding conics are transformed by reflection, rotation, translation, and dilation with versors in DCGA may be beneficial (e.g. in terms of numerical cost and accuracy of computation). Furthermore, DCGA has more general objects (cyclides) than conics, and differentiation is possible via simple commutator computations. But DCGA has no means to compute general intersections of conics, hence the use of CCGA becomes inevitable. Finally, extensions to three dimensions exist for (quadric) QCGA [1], and to any finite dimension for DCGA [6].

We expect that geometric algebra implementations like Gaalop [7], ganja.js [2] and The Clifford Multivector Toolbox (for MATLAB) [14] can compute with the geometric algebra for model we propose, including visualizations. 


\section{Acknowledgments}

Soli Deo Gloria ${ }^{8}$. The author EH requests the Creative Peace License[8] to be adhered to regarding the content of this work. Finally, we thank the anonymous reviewers for their helpful advice.

\section{References}

[1] S. Breuils, V. Nozick, A. Sugimoto, E. Hitzer, Quadric Conformal Geometric Algebra of $\mathbb{R}^{9,6}$, Adv. Appl. Clifford Algebras 28, No. 35 (2018), 1-16, https : / / do i . org / 10.1007/s00006-018-0851-1.

[2] S. De De Keninck, The Coffee Shop, https://enkimute.github.io/ganja. js / examples/coffeeshop. html, accessed 30 Aug. 2019.

[3] Desmos, Implicite functions online graphing software, https://www. desmos . com/calculator/pi5ofejgt0, accessed 02 Feb. 2019.

[4] L. Dorst, D. Fontijne, S. Mann, Geometric algebra for computer science, an objectoriented approach to geometry, Morgan Kaufmann, Burlington (2007).

[5] G. El Mir, C. Saint-Jean, M. Berthier, Conformal Geometry for Viewpoint Change Representation, Adv. Appl. Clifford Algebras 24(2), (2014), pp. 443-463, https: //doi.org/10.1007/s00006-013-0431-3.

[6] R. B. Easter, E. Hitzer, Double Conformal Geometric Algebra, Adv. Appl. Clifford Algebras 27(3) (2017), pp. 2175-2199, https://doi.org/10.1007/ s00006-017-0784-0.

[7] D. Hildenbrand, Introduction to Geometric Algebra Computing, 1st Edition, Chapman and Hall/CRC, Boca Raton (2018).

[8] E. Hitzer, The Creative Peace License, https : / gaupdate.wordpress . com/ 2011/12/14/the-creative-peace-license-14-dec-2011/.

[9] E. Hitzer, K. Tachibana, S. Buchholz, I. Yu, Carrier method for the general evaluation and control of pose, molecular conformation, tracking, and the like, Adv. Appl. Clifford Algebras 19(2) (2009), pp. 339-364, https://doi.org/10.1007/ s00006-009-0160-9.

[10] J. Hrdina, A. Navrat, P. Vasik, Geometric Algebra for Conics, Adv. Appl. Clifford Algebras (2018) 28:66, 21 pp., https://doi.org/10.1007/ s00006-018-0879-2.

[11] H. Li, Invariant Algebras and Geometric Reasoning, World Scientific, Singapore (2008).

[12] A. Naeve, L. Svensson, Projective Geometric Computing, The 5th International Conference on Clifford Algebras and their Applications in Mathematical Physics, IxtapaZihuatanejo, Mexico, June 27-July 4 (1999). Preprint: http: / kmr.nada.kth. se/papers /CVAP / ProjGeomComputing.pdf

[13] C. Perwass, Geometric Algebra with Applications in Engineering, Springer, Heidelberg (2008).

\footnotetext{
${ }^{8}$ J.C. Maxwell had inscribed at the gate of his Cavendish laboratory: The works of the Lord are great, Studied by all who have pleasure in them. [Psalm 111:2, New King James Version]. D. Capkova writes in Colloquium Comenius and Descartes, Foundation Comenius Museum, Naarden, 1997, p. 15, that: "Comenius' warning against onesided rationalism and against universal application of rationalism speaks to our time very urgently."
} 
[14] S. J. Sangwine, E. Hitzer, Clifford Multivector Toolbox (for MATLAB), Adv. Appl. Clifford Algebras 27(1) (2017), pp. 539-558, https://doi.org/10.1007/ s00006-016-0666-x.

[15] J. Zamora-Esquivel, $G_{6,3}$ Geometric Algebra; Description and Implementation, Adv. Appl. Clifford Algebras 24(2) (2014), pp. 493-514, https://doi.org/10. $1007 /$ s00006-014-0442-8.

\section{Eckhard Hitzer}

International Christian University,

Osawa 3-10-2, Mitaka-shi 181-8585, Tokyo, Japan

e-mail: hitzereicu.ac.jp

\section{Stephen J. Sangwine}

School of Computer Science and Electronic Engineering, University of Essex, Wivenhoe Park, Colchester, CO4 3SQ, United Kingdom.

e-mail: sjs@essex.ac.uk 